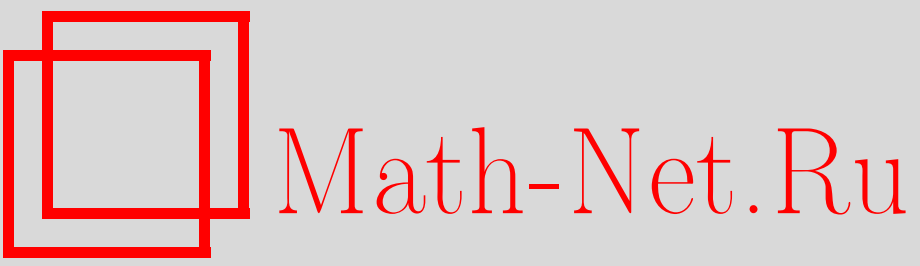

А. А. Кагарманов, О несуществовании полинома, восстанавливающего кольцо функций по алгебре Ли векторных полей над полями положительной характеристики, УМH, 1998, том 53, выпуск 1, 217-218

DOI: https://doi.org/10.4213/rm24

Использование Общероссийского математического портала Math-Net.Ru подразумевает, что вы прочитали и согласны с пользовательским соглашением

http://www.mathnet.ru/rus/agreement

Параметры загрузки:

IP : 54.89 .56 .158

26 апреля 2023 г., $17: 25: 48$ 


\title{
О НЕСУЩЕСТВОВАНИИ ПОЛИНОМА, ВОССТАНАВЛИВАЮЩЕГО КОЛЬЦО ФУНКЦИЙ ПО АЛГЕБРЕ ЛИ ВЕКТОРНЫХ ПОЛЕЙ НАД ПОЛЯМИ ПОЛОЖИТЕЛЬНОЙ ХАРАКТЕРИСТИКИ
}

\author{
А. А. КАГАРМАНОВ
}

Пусть $W_{n}=\operatorname{der} E_{n}$ - алгебра Ли дифференцирований кольца многочленов $E_{n}=$ $K\left[x_{1}, \ldots, x_{n}\right]$ от $n$ коммутирующих переменных. Алгебру $W_{n}$ можно рассматривать как

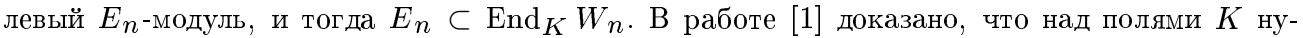
левой характеристики существует такой ассоциативньй полином $f\left(t_{1}, \ldots, t_{l}\right)$, что для любых $g_{1}, \ldots, g_{l} \in W_{n}$ значение $f\left(\operatorname{ad}\left(g_{1}\right), \ldots, \operatorname{ad}\left(g_{l}\right)\right) \in E_{n}$ и отображение $f:\left(\operatorname{ad} W_{n}\right)^{\otimes l} \rightarrow E_{n}$ является эпиморфизмом.

Этот полином позволяет достаточно конструктивно восстанавливать коммутативную алгебру бесконечно дифференцируемых функций гладкого $n$-мерного многообразия по алгебре Ли его векторных полей. Такие полиномы мы будем называть восстанавлив ающими полиномами. Цель данной заметки - доказать, что без дополнительных ограничений на поля положительной характеристики этот резултат не переносится. Нами получена следующая

ТеОрема. Если char $K=2$, то полинома $f$, восстанавливающего по алгебре Ли $W_{1}$ специальных дифференцирований кольца $E_{1}=O[x]$ саму алгебру $E_{1}$, не существует.

Определения специальных дифференцирований в случае полей положительной характеристики можно посмотреть в [2].

ЛЕмма 1. Для любого натурального $m$ векторное подпространство $H_{m}$, натянутое на $\left\langle e_{-1}, e_{0}, \ldots, e_{p^{m}-2}\right\rangle$ в алгебре $W_{1}$ над полем характеристики $p$, является подалгеброй в $W_{1} ;$ здесь $e_{k}=x^{(k+1)} \partial_{x}-$ стандартный базис в $W_{1}$.

Рассмотрим подалгебру в $O[x]$ - алгебре разделенных степеней вида $O_{m}(x)=\left\langle 1, x, x^{(2)}, \ldots\right.$, $\left.x^{\left(p^{m}-1\right)}\right\rangle_{K}$; это подалгебра, так как $x^{(k)} \cdot x^{(l)}=\left(\begin{array}{c}k+l \\ k\end{array}\right) x^{(k+l)}=0$, если $k+l \geqslant p^{m}, k, l<p^{m}$. Это следует из того, что если рассматривать целую функцию $\tau_{p}(n)$ - максималњную степень числа $p$, на которую делится $n$ !, то

$$
\tau_{p}(n)=\sum_{k=1}^{\infty}\left[\frac{n}{p^{k}}\right]
$$

где $[x]$ - целая часть числа $x$. Поэтому максимальная степень числа $p$, на которую в данном случае делится $\left(\begin{array}{c}k+l \\ k\end{array}\right)$, очевидно, есть $\tau_{p}(k+l)-\tau_{p}(k)-\tau_{p}(l)=\sum_{k=1}^{m}\left(\left[\frac{k+l}{p^{i}}\right]-\left[\frac{k}{p^{i}}\right]-\left[\frac{l}{p^{i}}\right]\right)$. Каждое слагаемое в этой сумме неотрицателшно, так как $[x+y] \geqslant[x]+[y]$, а последнее слагаемое $(i=m)$ равно 1 , поэтому в нашем поле $K$ биномиальный коэффициент $\left(\begin{array}{c}k+l \\ k\end{array}\right) \equiv 0$. Очевидно, что алгебра дифференцирований $O_{m}(x)$ есть $H_{m}(x)$, что и требовалось.

Лемма 2. Если char $K=2$, то в $W_{1}$ подалгебры $H_{m}$ из леммы 1 содержат идеаль $I_{m}$ вида $I_{m}=\left\langle e_{-1}, e_{0}, \ldots, e_{2^{m}}-3\right\rangle_{K}$ и только такие.

Пусть в $H_{m}$ есть идеал $I$ и ненулевой элемент $\omega=\sum_{k=-1}^{N} a_{k} e_{k} \in I$. В силу $\left[e_{k}, e_{-1}\right]=$ $e_{k-1}$ получаем следующие соотношения:

$$
\begin{aligned}
& {\left[e_{-1}, \omega\right]=\sum_{k=-1}^{N-1} a_{k+1} e_{k} \in I,} \\
& {[\underbrace{e_{1}, \ldots, e_{-1}}_{s}, \omega]=\sum_{k=-1}^{N-s} a_{k+s} e_{k} \in I,}
\end{aligned}
$$


где $s=1, \ldots, N+1$. Из этой треугольной относительно $e_{-1}, \ldots, e_{N}$ системы получаем, что $\left\{e_{-1}, e_{0}, \ldots, e_{N}\right\}_{K} \in I$. Далее, учитьвая, что $H_{m}=\left\langle e_{-1}, e_{0}, \ldots, e_{2}{ }_{-2}\right\rangle_{K}$, получаем, что $\left\langle e_{-1}, e_{0}, \ldots, e_{2^{m}-3}\right\rangle_{K} \in I$. Осталось показать, что $I_{m}=\left\langle e_{-1}, e_{0}, \ldots, e_{2^{m}}-3\right\rangle_{K}$ - идеал в $H_{m}$. Для этого достаточно показать, что для любых положительных целых $q$ и $l$ таких, что $q+l=2^{m}-2$, выполняется равенство $\left[e_{q}, e_{l}\right]=0$. Это следует из того, что все $\left(\begin{array}{c}2^{m} \\ i\end{array}\right)$ четны, а значит, все $\left(2^{\frac{2}{i}-1}\right)$ нечетны, поэтому

$$
\left[e_{q}, e_{l}\right]=\left(\left(\begin{array}{c}
q+l+1 \\
q+1
\end{array}\right)-\left(\begin{array}{c}
q+l+1 \\
l+1
\end{array}\right)\right) \cdot e_{q+l}=\left(\left(\begin{array}{c}
2^{m}-1 \\
q+1
\end{array}\right)-\left(\begin{array}{c}
2^{m}-1 \\
l+1
\end{array}\right)\right) \cdot e_{2}{ }^{m-2}=0
$$

над нашим полем $K$. Лемма доказана.

Лемма 3. Пусть сhar $K=2, \quad A-$ любая конечномерная подалгебра вида $\left\langle 1, x, x^{(2)}, \ldots, x^{(l)}\right\rangle_{K}$ в $O[x]$. Тогда $l+1$ есть степень числа 2.

Если $l+1$ - степень числа 2 , то получаем лемму 1 . Обратно, если $A$ - подалгебра, то для $k=1, \ldots, l$ выполняется $0=x^{(k)} \cdot x^{(l+1-k)}=\left(\begin{array}{c}l+1 \\ k\end{array}\right) \cdot x^{(l+1)}$, поэтому в треугольнике Паскаля $(l+1)$-я строка $\left(\begin{array}{c}l+1 \\ k\end{array}\right)(k=1, \ldots, l)$ состоит из четных чисел. Покажем, что $n=l+1$-степень числа 2. Представим $n=2^{k}+r, 0 \leqslant r<2^{k}$. Пусть $r>0$. Если все $\left(\begin{array}{c}n \\ i\end{array}\right)(i=1, \ldots, n-1)$ четны, то над $\mathbb{Z}_{2}$

$(1+x)^{n}=1+x^{n}=(1+x)^{2^{k}} \cdot(1+x)^{r}=\left(1+x^{2^{k}}\right)\left(1+r x+\cdots+x^{r}\right)=1+\cdots+x^{r}+\cdots+x^{n}$.

Противоречие с тем, что $0<r<2^{k}$. Лемма доказана.

Перейдем теперь к доказательству теоремы. Пусть существует полином $f$, осуществляющий ненулевое отображение $f:\left(\operatorname{ad} W_{1}\right)^{\otimes} \rightarrow O_{1}[x]$. Тогда существует набор $e_{i_{1}}, \ldots, e_{i_{l}}$ из стандартного базиса $W_{1}$ такой, что $f\left(\operatorname{ad}\left(e_{i_{1}}\right), \ldots, \operatorname{ad}\left(e_{i_{l}}\right)\right) \neq 0$. Выберем натуральное $m$ таким, что $e_{i_{1}}, \ldots, e_{i_{l}} \in H_{m}$ из леммы 1 . Тогда отображение $\left.f\right|_{H_{m}}$ - ненулевое. Поэтому, если обозначить через $A$ линейное пространство в $\operatorname{End}_{k} H_{m}$, порожденное $f\left(\operatorname{ad} \omega_{1}, \ldots, \operatorname{ad} \omega_{l}\right)$, то $H=A \cap O(x)$ - ненулевая подалгебра в $\operatorname{End}_{k} H_{m} \cap O(x)$. Более того, в силу

$$
\left[g\left(\operatorname{ad} \omega_{1}, \ldots, \operatorname{ad} \omega_{l}\right), \operatorname{ad} \vartheta\right]=\left.\sum_{i=1}^{l} g\right|_{t_{i}=\left[\omega_{i}, \vartheta\right]} ^{t_{k}=\omega_{k}, k \neq i}
$$

вьполняется включение

$$
\left[\operatorname{ad} H_{m}, H\right] \subset H \text {. }
$$

В силу того, что $H$ - ненулевая подалгебра и выполняется (2), $H$ ○ $\partial$ выдерживает действие $e_{-1}, e_{0}, e_{1}, \ldots$, т.е.

$$
\left[\operatorname{ad} e_{k}, H \circ \partial\right] \subset H \circ \partial .
$$

С другой стороны, $H \circ \partial$ конечномерна, и, более того, так как $H$ - подалгебра, то из леммы 3 , которую можно применять в силу того, что $H \circ \partial$ имеет вид $\left\langle e_{-1}, e_{0}, \ldots, e_{N}\right\rangle_{K}$ и $H=\left\langle 1, x, x^{(2)}, \ldots, x^{\left(2^{m_{1}}-1\right)}\right\rangle_{K}$, следует $\operatorname{dim}_{K} H=2^{m_{1}}$. Очевидно, что $m_{1} \leqslant m$. В силу (3) $H \circ \partial$ содержит $e_{-1}, e_{0}, \ldots, e_{2^{m}}-3$, а значит, $m_{1}=m$ и $H \circ \partial=H_{m}$. С другой стороны, $H \circ \partial \subseteq I_{m} \not \subset$, так как $\partial \in I_{m}$ и $H$ порождена мономами $\operatorname{ad} \omega_{1} \cdot \ldots \cdot \operatorname{ad} \omega_{l}$, т.е. $H \circ \partial$ лежит в идеале $I_{m}$. Противоречие. Теорема доказана.

\section{СПИСОК ЛИТЕРАТУРЫ}

[1] Размыслов Ю. П. // Матем. сб. 1983. Т. 112. № 1. С. 97-125. [2] Кострикин А. И., Шафоревич И. Р. // Изв. АН СССР. Сер. матем. 1969. Т. 33. № 2. С. 251-322. 